\title{
Addendum to Strangeness Production and Color Deconfinement团
}

\author{
P. Castorina ${ }^{\mathrm{a}, \mathrm{b}}$, S. Plumari ${ }^{\mathrm{a}, \mathrm{b}}$ and H. Satz ${ }^{\mathrm{c}}$ \\ a: Dipartimento di Fisica ed Astronomia, Universitá di Catania, Italy \\ b: INFN, Sezione di Catania, Catania, Italy \\ c: Fakultät für Physik, Universität Bielefeld, Germany
}

\begin{abstract}
:
Recent extensive data from the beam energy scan of the STAR collaboration at BNLRHIC provides the basis for a detailed update for the universal behavior of the strangeness suppression factor $\gamma_{s}$ as function of the initial entropy density, as proposed in our recent paper [1].
\end{abstract}

This note presents an extension of our recent work on strangeness suppression in hadronic and nuclear collisions [1]. We had shown there that the suppression factor $\gamma_{s}$, needed in hadron resonance gas studies to account for the production of strange hadrons in $p p, p A$ and $A A$ collisions at low and intermediate energies, becomes a universal function in terms of the initial entropy density or the initial temperature. Recently, extensive data for hadron production at various energies and as function of centrality were obtained by the beam energy scan (BES) of the STAR experiment at RHIC [2], and the analysis of these data has provided values for $\gamma_{s}$ over a considerable range of kinematic variables. We have expressed these $\gamma_{s}$ values, given in the experimental analysis as functions of the collision energy and the centrality, in terms of the initial entropy density of the corresponding collisions. This is shown to provide an excellent and more detailed support of our claim that in such a description all $p p, p A$ and $A A$ data fall on one universal curve, which varies from about 0.5 to unity precisely in the color deconfinement transition region.

The mentioned BES study of STAR analyses hadron production for Au-Au collisions at cms energies of 7.7, 11.5, 19.627 and $39 \mathrm{GeV}$, in each case as function of centrality as measured by the number of participating nucleons, $N_{\text {part }}$, from peripheral to central. The data are subjected to resonance gas analyses based on grand canonical and canonical (exact strangeness conservation) formulations, fitting either overall yields or yield ratios. As a result of these analyses, values of $\gamma_{s}$ are provided for $A u-A u$ collisions as function of $\sqrt{s}$ and centrality. In our analysis we use the canonical formulation for the abundance ratios; however, the other forms do not vary greatly. Our input, as taken from the experimental study, thus starts from the given values of $\gamma_{s}\left(\sqrt{s}, N_{\text {part }}\right)$ for Au-Au collisions.

${ }^{*}$ P. Castorina, S. Plumari and H. Satz, Int. J. Mod. Phys. E26 (2017) 1750081 (arXiv:1709.02706) 
The initial entropy density $s_{0}$ is given in the one-dimensional hydrodynamic formulation [3] by the form

$$
s_{0} \tau_{0} \simeq \frac{1.5\left(N_{\mathrm{part}}^{x} / 2\right)}{\pi R_{x}^{2}}\left(\frac{d N_{\mathrm{ch}}^{x}}{d y}\right)_{y=0}^{x}, \text { with } x \sim p p, p A, A A .
$$

Here $\left(d N^{x} / d y\right)_{y=0}$ denotes the number of produced charged secondaries, normalized to half the number of participants $N_{\text {part }}^{x}$, in reaction $x$. This form is chosen in multiplicity analyses [4] in order to obtain $\left(N_{\text {part }}^{x} / 2\right)=1$ for $p p$ and $\left(N_{\text {part }}^{x} / 2\right)=A$ for central $A A$ interactions. The result is shown in Fig. 1, The fits indicated there are provided by [4]; the details are indicated also in [1].

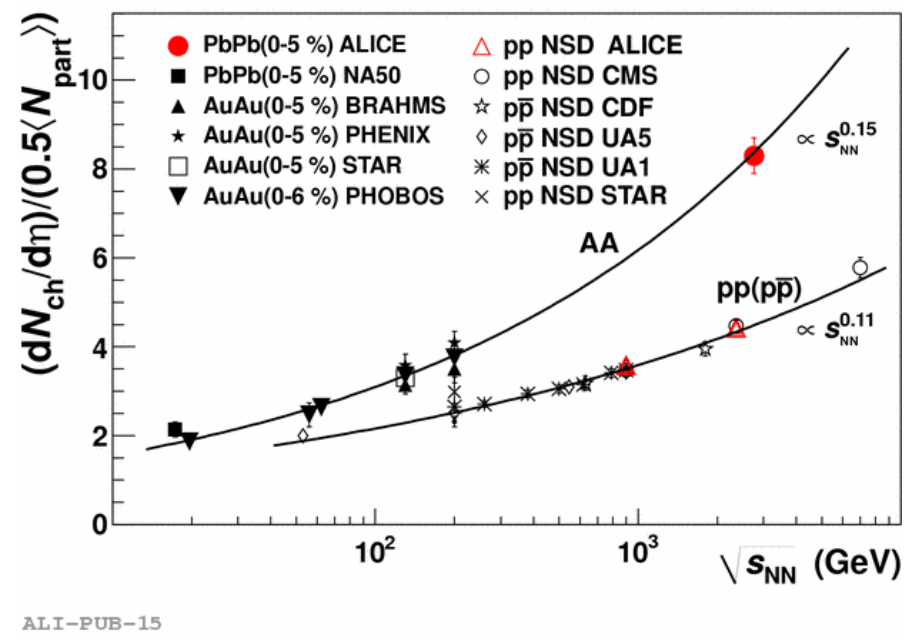

Figure 1: Average charged multiplicities in $p p$ and $A A$ collisions as function of cms energy $\sqrt{s}[4]$.

Using this information, we now want to convert the values of $\gamma_{s}\left(\sqrt{s}, N_{\text {part }}\right)$ into $\gamma_{s}\left(s_{0} \tau_{0}\right)$. This requires a specification of the transverse area in the different kinematic situations. As in [1], we use $R_{p p}=0.8 \mathrm{fm}$ and $R_{p P b}=R_{p}\left(0.5 N_{\text {part }}\right)^{1 / 3}=1.3 \mathrm{fm} \mathrm{[5}$, 6]; for the centrality dependent $A u-A u$ collisions, we use $R_{\text {AuAu }}=1.25\left(N_{\text {part }} / 2\right)^{1 / 3}$, generalizing the form $R_{A A}=1.25 A^{1 / 3}$ for central $A A$ collisions to non-central interactions. This is evidently only a rough estimate; in a more detailed future study, we will determine the transverse area by means of a Glauber analysis.

With the transverse areas thus specified, we can now determine the strangeness suppression factor $\gamma_{s}$ as function of $s_{0} \tau_{0}$ for the results of the STAR BES experiments, together with the $p p$ and $p P b$ values already used in [1]. The result is shown in Fig. 2, with $s_{0} \tau_{0}$ in units $\mathrm{fm}^{-2}$. In the bottom display, we use a logarithmic scale for $s_{0} \tau_{0}$, in order to show the transition region in more detail. In both cases, the solid curve shown is

$$
\gamma_{s}=1-0.997 \exp \left\{-0.575 s_{0} \tau_{0}\right\}
$$

it is meant just to guide the eye and is not a best fit. In [1], we had emphasized that the disappearance of strangeness suppression coincides with the onset of deconfinement. To see this here as well, we recall that lattice studies [8] give $T_{c}=154 \pm 9 \mathrm{MeV}$ for the 
deconfinement temperature; the corresponding range for the entropy density is given by $s_{0}=2.5 \pm 0.3 \mathrm{fm}^{-3}$. With the conventional choice of $\tau_{0} \simeq 1 \mathrm{fm}$ for the thermalization time, Fig. 2 corroborates nicely the coincidence of deconfinement and strangeness equilibration.
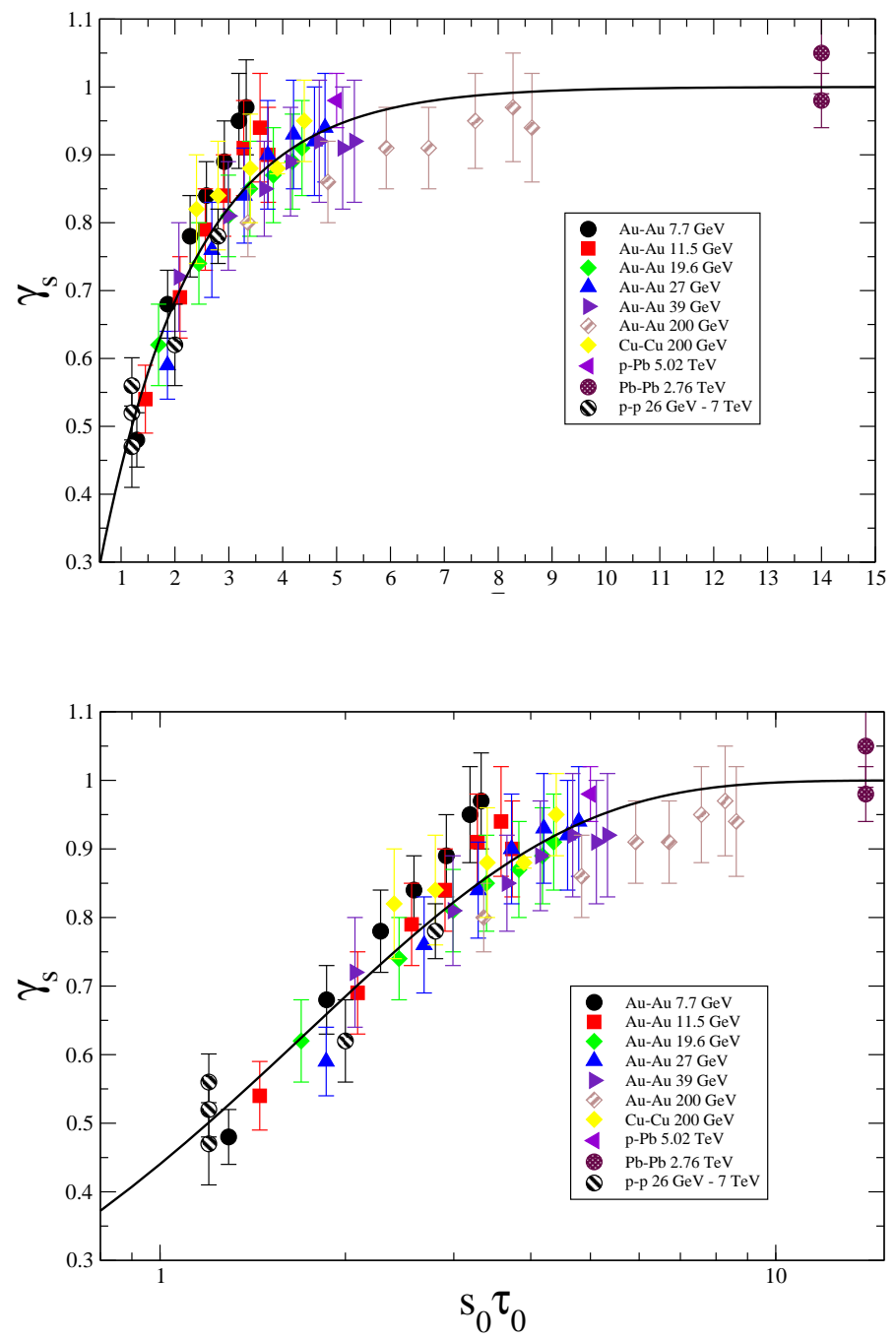

Figure 2: Strangeness suppression factor $\gamma_{s}$ as function of $s_{0} \tau_{0}$ for $p p, p P b$ [7] and $A u-A u$ collisions 22 at different collision energies and centralities. For the solid curve, see text.

From Fig. 2 we see moreover that the STAR data at all considered collision energies lead for the most central collisions to values of $\gamma_{s}$ in the range $0.9-1.0$. The transition to lower values of $\gamma_{s}$ sets in as the collisions become more peripheral, with lower $s_{0} \tau_{0}$. For this reason, various earlier data [9] restricted to central collisions had concluded that $\gamma_{s} \simeq 1$. To obtain a complete picture of strangeness suppression in $A A$ collisions and its relation to $p p$ interactions, it thus seems necessary to include peripheral $A A$ collisions as well. 


\section{Acknowledgements:}

We are grateful to $\mathrm{Nu} \mathrm{Xu} \mathrm{(LBL} \mathrm{Berkeley/Wuhan)} \mathrm{for} \mathrm{calling} \mathrm{the} \mathrm{STAR} \mathrm{data} \mathrm{to} \mathrm{our}$ attention. Paolo Castorina gratefully acknowledges support by the Deutsche Forschungsgemeinschaft (DFG) through the grant CRC-TR 211, "Strong interaction matter under extreme conditions".

\section{References}

[1] P. Castorina, S. Plumari and H. Satz, Int. J. Mod. Physics E26 (2017) 1750081 (arXiv:1709.02706 [nucl-th])

[2] L. Adamczyk et al. (STAR Coll.), Phys. Rev. C96 (2017) 044904, (arXiv:1701.07065 [nucl-ex])

[3] J. D. Bjorken, Phys. Rev. D27 (1983) 140.

[4] K. Aamodt et al. (ALICE Coll.), Phys. Rev. Lett. 105 (2010) 252301.

[5] B. Abelev et al. (ALICE Coll.), Phys. Rev. Lett. 110 (2013) 052301.

[6] P. Castorina, S. Plumari and H. Satz, Int. J. Mod. Physics E25 (2016) 1650058 (arXiv:1603.06529 [hep-ph])

[7] For the fits of the $p p$ and $p P b$ data used, see ref. [1].

[8] A. Bazazov et al. (HotQCD), Phys. Rev. D90 (2014) 094503.

[9] For a summary of previous data, see

A. Andronic, P. Braun-Munzinger and J. Stachel, Nucl. Phys. A772 (2006) 167. 